\title{
Impact of the number of conditioning pulses on motor cortex excitability: a transcranial magnetic stimulation study
}

\author{
Petyo Nikolov ${ }^{1,2}$. Johanna V. Zimmermann ${ }^{1} \cdot$ Shady S. Hassan $^{3} \cdot$ Philipp Albrecht $^{2} \cdot$ Alfons Schnitzler $^{1,2}$. \\ Stefan J. Groiss ${ }^{1,2}$ (D)
}

Received: 28 July 2020 / Accepted: 7 December 2020 / Published online: 29 December 2020

(c) The Author(s) 2020

\begin{abstract}
Conditioning transcranial magnetic stimulation (TMS) with subthreshold conditioning stimulus followed by supra-threshold test stimulus at inter-stimulus intervals (ISI) of $1-5 \mathrm{~ms}$ results in inhibition (SICI), while ISI at 10-15 ms results in facilitation (ICF). One concerning issue, applying ICF/SICI protocols on patients is the substantial protocol variability. Here, we hypothesized that increasing the number of CS could result in more robust ICF/SICI protocols. Twenty healthy subjects participated in the study. Motor-evoked potentials (MEP) were obtained from conditioning TMS with a varying number of conditioning stimuli in 3, 4, 10, and $15 \mathrm{~ms}$ ISI over the primary motor cortex. MEP amplitudes were then compared to examine excitability. TMS with 3, 5, and 7 conditioning stimuli but not with one conditioning stimulus induced ICF. Moreover, $10 \mathrm{~ms}$ ISI produced stronger ICF than $15 \mathrm{~ms}$ ISI. Significant SICI was only induced with one conditioning stimulus. Besides, $3 \mathrm{~ms}$ ISI resulted in stronger SICI than $4 \mathrm{~ms}$ ISI. Only a train of conditioning stimuli induced stable ICF and may be more advantageous than the classical paired pulse ICF paradigm.
\end{abstract}

Keywords Transcranial magnetic stimulation $\cdot$ Facilitation $\cdot$ Inhibition $\cdot$ Conditioning stimuli

$\begin{array}{ll}\text { Abbreviations } \\ \text { AMT } & \text { Active motor threshold } \\ \text { FDI } & \text { First dorsal interosseus } \\ \text { CS } & \text { Conditioning stimulus; } \\ \text { EPSP } & \text { Excitatory post-synaptic potential } \\ \text { ICF } & \text { Intracortical facilitation } \\ \text { MEP } & \text { Motor-evoked potential }\end{array}$

Communicated by Winston D Byblow.

Petyo Nikolov and Johanna V. Zimmermann have contributed equally to this work.

Supplementary Information The online version contains supplementary material available at https://doi.org/10.1007/s0022 1-020-06010-7.

Stefan J. Groiss

groiss@uni-duesseldorf.de

1 Institute of Clinical Neuroscience and Medical Psychology, Heinrich Heine University Düsseldorf, Düsseldorf, Germany

2 Department of Neurology, Medical Faculty, Heinrich Heine University Düsseldorf, Düsseldorf, Germany

3 Department of Neurology, Medical Faculty, Assiut University Hospital, Assiut, Egypt
MPO Maximal stimulator output

SICI Short-interval intracortical inhibition

STDP Short-term dependent plasticity

TMS Transcranial magnetic stimulation

TS Test stimulus

\section{Introduction}

Transcranial magnetic stimulation (TMS) is an established and painless non-invasive method to study motor cortex physiology. The motor-evoked potential (MEP) amplitude can be used as marker for corticospinal excitability. Conditioning TMS is a paradigm, where the test stimulus (TS) is preceded by conditioning stimuli (CS). In this relation, several conditioning TMS techniques to study inhibitory and excitatory mechanisms have been developed. One of them is the classical paired pulse technique including short-interval intracortical inhibition (SICI) with inter-stimulus intervals (ISI) of 1-5 ms (Kujirai et al. 1993), as well as intracortical facilitation (ICF) with 10-15 ms ISI (Ilić et al. 2002). Both techniques implement one subthreshold single CS, followed by one supra-threshold TS. 
ICF is thought to originate from excitatory interneurons and to be glutamate-dependent (Lazzaro et al. 1999; Ilić et al. 2002). Although ICF is considered a cortical phenomenon, additional spinal mechanisms cannot be fully excluded (Lazzaro et al. 2006). SICI, on the other hand, is viewed as pure intracortical phenomenon, which is probably based on monosynaptic inhibitory output toward the first motor neuron. It seems to be generated by low-threshold GABA-interneurons at the synaptic level (Ilić et al. 2002; Lazzaro et al. 2006).

ICF/SICI protocols have been implemented in clinical research, to determine glutamate/GABA homeostasis impairment in multiple neurological or psychiatric disorders, and already contributed to better understanding of the pathomechanisms behind some of them. However, ICF/SICI protocols can exhibit considerable outcome variability (Orth et al. 2003) and further research to increase reliability is desirable, as both techniques generally yield the potential for biomarkers (Berardelli et al. 2008; Doruk Camsari et al. 2019).

In this context, Hanajima et al. developed a protocol called triad conditioning facilitation (TCF), using three CS which was supposed to entrain the intrinsic rhythm of the motor cortex and thus strengthen the induced facilitation (Hanajima et al. 2009). Recently, we have shown that TCF could share the same mechanism of ICF, and the intensity of both CS and TS can modulate the degree of facilitation (Hassan et al. 2020). However, not only TMS intensity, but also the number of CS could modulate the degree of facilitation. Thus, in the current study, we hypothesized that increasing the number of CS could strengthen ICF.

\section{Materials and methods}

\section{Participants}

Twenty healthy participants (10 males; 10 females, three left-handed, mean age $24.4 \pm 1.8$ years) were enrolled in the study. All participants provided written informed consent in accordance with the Declaration of Helsinki (World Medical Association 2013) and the Ethical Committee of the Medical Faculty, Heinrich Heine University Düsseldorf prior to participation (Study ID: 5738R). Exclusion criteria were contraindication to TMS (e.g., due to metallic and/or magnetic implants), severe intestinal, neurological, or psychiatric diseases, the use of any medication acting on the central nervous system (e.g., benzodiazepines, anti-epileptic, and/or psychotropic drugs), blood clotting dysfunction, pregnancy, and diagnosed peripheral/retinal neuropathy.

\section{Transcranial magnetic stimulation}

TMS was applied by a Magstim ${ }^{\mathrm{TM}}$ magnetic stimulator (The Magstim Co. Ltd, Whitland, UK) through a figure-of-eight coil. Eight magnetic stimulators were connected with a specially designed combining module (The Magstim Co. Ltd., Whitland, UK) to allow the application of a burst with up to eight monophasic magnetic stimuli through a single coil. The coil was placed above the primary motor cortex (M1) of the dominant hemisphere and over the individual hotspot for the first dorsal interosseus muscle (FDI). During stimulation, the coil was always positioned tangentially to the scalp with the handle pointing backwards and laterally at a $45^{\circ}$ angle to the sagittal plane. In this way, a posterior-anterior current direction in the brain was ensured (Rothwell 1997). The configuration aims to trans-synaptically activate the corticospinal system by means of horizontal cortico-cortical connections (Lazzaro et al. 2004). After determination of the individual TMS hotspot, the active motor threshold (AMT) was defined as the lowest stimulation intensity that evoked a response of at least $100 \mu \mathrm{V}$ during $5-10 \%$ maximal contraction of FDI in at least 5 of 10 trials using the relative frequency method (Rossini et al. 2015). While ICF was studied with 10 and 15 ms ISI (Kujirai et al. 1993), SICI was studied with 3 and 4 ms ISI (Lazzaro et al. 2006). For each ISI, the number of CS was varied between 1, 3, 5, and 7. Here, the ISI between multiple CS and between CS and TS were always the same within one condition. Single-pulse TS without CS was used as control condition. While stimulation intensity of $90 \%$ AMT was applied for CS, intensity resulting in $0.5 \mathrm{mV}$ MEP response was set for TS. The order of stimulation conditions was randomized within subjects and applied in a shuffled order.

\section{Electromyographic recording}

EMG signals were recorded from the FDI muscle with disposable $\mathrm{Ag}-\mathrm{AgCl}$ surface electrodes $(20 \times 15 \mathrm{~mm}$, Ambu, Denmark). The active electrode was placed on the muscle belly, whereas the reference was located over the base of the metacarpophalangeal joint of the index finger. EMG signals were amplified (Digitimer D360, Digitimer Ltd, Hertfordshire, UK), band passed between 10 and $5 \mathrm{kHz}$, digitized at a sampling rate of $5 \mathrm{kHz}$, and stored on a desktop computer for off-line analysis.

\section{Experimental design}

Participants were seated in a comfortable reclining chair with arms placed on cushioned armrests during the entire experiment. Subsequently, electromyographic electrodes 
were attached to the FDI. The individual hot spot was determined in steps of $0.5-1 \mathrm{~cm}$, starting $5 \mathrm{~cm}$ lateral and $1.5 \mathrm{~cm}$ anterior of the vertex, and defined as the spot producing the largest MEP amplitudes. The hotspot was then marked directly on the scalp with a soft-tip pen to insure constant placement of the TMS coil throughout the session. TMS intensities for AMT, as well as for the triggering of $0.5 \mathrm{mV}$ MEP were determined once at the beginning and were then used throughout the experiment. To ensure subject compliance and maintain similar level of attention, MEP recordings of ICF, SICI, and single-pulse MEP were splitted into four blocks. The four blocks consisted of the following configurations:

(I) 1 CS -3 CS MEP in 10-15 ms ISI

(II) 5 CS- 7 CS MEP in 10-15 ms ISI

(III) 1 CS-3 CS MEP in 3-4 ms ISI

(IV) 5 CS-7 CS MEP in 3-4 ms ISI.

Here, single-pulse MEP was measured separately in each block, which means that each block had a separate control condition. 20 MEPs were recorded for each condition (including 20 single-pulse control MEPs for each block).

Through the course of the entire experiment, muscle relaxation was monitored by an oscilloscope (Rigol DS1074B, Hirschau, Germany). Subjects were also instructed to look at a fixation cross centered in front of them and silently count the number of bursts applied to maintain similar level of attention.

\section{Data analysis and statistical evaluation}

EMG data were analyzed with Signal Software (Cambridge Electronic Design, Cambridge, UK). Trials were visually inspected. Trials showing voluntary EMG activity immediately before the TMS pulse, as well as trials where no TMS pulse was presented due to technical reasons, were rejected from the analysis (mean $=1.3$ rejected trials per condition). MEP with atypical forms and latencies were visually controlled for and not observed in our experiments, so that no MEP response with CS alone could be elicited, even with higher CS intensities (Hassan et al. 2020). Maximum peakto-peak MEP amplitudes were determined for each trial. Subsequently, peak-to-peak MEP amplitudes were averaged over all trials of each condition. Then, MEP ratios were defined as ratio between conditioned MEP and single-pulse MEP. All MEP amplitudes were logarithmically transformed for further analysis.

Statistical evaluation was performed with SPSS. Shapiro-Wilk test was used to test for normality. Two-way repeated ANOVA was used to compare MEP ratios separately for facilitation with the factors number of CS $(1,3$,
5, $7 \mathrm{CS}$ ) and ISI (10 and $15 \mathrm{~ms}$ ), and for inhibition with the factors CS (1, 3, 5, $7 \mathrm{CS}$ ) and ISI (3 and $4 \mathrm{~ms})$, respectively.

To determine the degree of MEP change of conditioning TMS compared to single-pulse TMS, absolute MEP amplitudes for each ISI were compared using one-way ANOVA (10 and $15 \mathrm{~ms}$ ISI) and Friedman test ( 3 and $4 \mathrm{~ms}$ ISI), respectively. For each ISI, two separate ANOVAs or Friedman tests were conducted (for 1-3 CS and 5-7 CS, respectively) in order take into account the four different blocks, since each of them had their own single-pulse control condition. If applicable, either Bonferroni or Dunn test was carried out for post hoc analysis. To confirm comparability of the single-pulse control conditions, the single-pulse MEP amplitudes for each block were compared with one-way ANOVA.

\section{Results}

\section{AMT and TS intensities}

Mean AMT intensity was $37 \% \pm 2$ SEM of maximal stimulator output (MPO), and mean CS intensity was $33 \% \pm 2$ SEM of MSO. Furthermore, mean TS intensity was $53 \% \pm 3$ SEM of MSO.

\section{Facilitation (10 and 15 ms ISI)}

Two-way ANOVA revealed significant main effects of CS number and ISI for MEP ratios, with no interaction between the main effects. MEP ratios significantly differed between number of CS $(F=10.73, p=0.001)$. Compared to $1 \mathrm{CS}$, (mean $=1 \pm 0.07$ SEM), MEP ratios resulting from $3 \mathrm{CS}$ $($ mean $=1.9 \pm 0.2 \mathrm{SEM} ; p<0.001), 5 \mathrm{CS}($ mean $=2.1 \pm 0.2$ SEM; $p=0.004)$, and 7 CS $($ mean $=1.9 \pm 0.1$ SEM; $p=0.014)$ were larger. There was no difference between 3 CS, 5 CS and 7 CS MEP ratios ( $p>0.05$ for all three), (Fig. 1a). Furthermore, MEP ratios significantly differed between ISI $(F=5.46 ; p=0.031) .10 \mathrm{~ms}$ ISI $($ mean $=1.9 \pm 0.1 \mathrm{SEM})$ resulted in larger MEP ratio than $15 \mathrm{~ms}$ ISI (mean $=1.6 \pm 0.1 \mathrm{SEM} ; p<0.05$ ). For a summary of mean MEP amplitudes between conditions, please see Supplementary Table 1, Facilitation.

\section{Inhibition (3 and 4 ms ISI)}

Two-way ANOVA revealed significant main effects of CS number and ISI over MEP ratios, with no interaction between the main effects. MEP ratios significantly differed between number of CS $(F=18.43, p<0.001) .1 \mathrm{CS}$ MEP ratio (mean $=0.7 \pm 0.07 \mathrm{SEM}$ ) was significantly smaller than 5 CS (mean $=1.6 \pm 0.2$ SEM, $p=0.003)$ and 7 CS (mean $=1.9 \pm 0.3$ SEM, $p<0.001)$ MEP ratios. Also, 

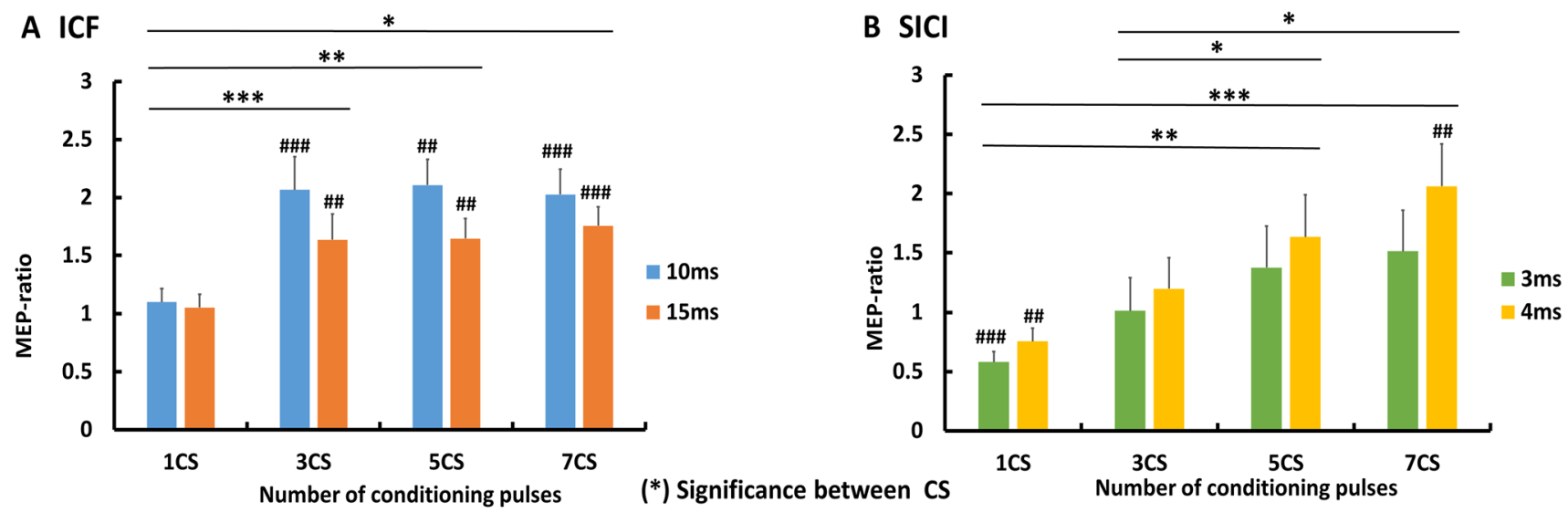

(\#) Significance to single pulse

Fig. 1 a Conditioning TMS with 10-15 ms (ICF); b conditioning TMS with 3-4 ms (SICI). Comparison between conditioning TMSMEP ratios with variable number of conditioning stimuli. Significant differences between MEP ratios are marked with asterisk. Significant

3 CS MEP ratio (mean $=1.1 \pm 0.1 \mathrm{SEM}$ ) was significantly smaller than 5 CS $(p=0.014)$ and 7 CS $(p=0.004)$ MEP ratios. There was no difference between 1 and 3 CS MEP ratios $(p>0.05)$, as well as between 5 and 7 CS MEP ratios $(p>0.05)$ (Fig. 1b). Furthermore, MEP ratios significantly differed between ISI $(F=24.84, p<0.001) .3 \mathrm{~ms}$ ISI (mean $=0.9 \pm 0.1 \mathrm{SEM})$ resulted in significantly smaller MEP ratio than $4 \mathrm{~ms}$ ISI (mean $=1.5 \pm 0.2 \mathrm{SEM}, p<0.001$ ). For a summary of mean MEP amplitudes, please see Supplementary Table 1 , Inhibition.

\section{Discussion}

Our study has three main findings. First, a train of CS strengthens ICF, which is induced more effectively with 10 ms ISI. Second, a train with more than 3 CS do not offer additional benefit. Third, a train of CS reduces SICI, which is induced more effectively with $3 \mathrm{~ms}$ ISI and $1 \mathrm{CS}$.

Our results are consistent with earlier reports on the topic, where a train of 3 CS alleviated SICI with $3 \mathrm{~ms} \mathrm{ISI} \mathrm{and}$ enhanced ICF with $10 \mathrm{~ms}$ ISI (Calancie et al. 2018).

\section{Facilitation (10 and 15 ms ISI)}

To our knowledge, this is the first study, where a varying number of CS was applied in an ICF protocol. We could show that a train of CS strengthens facilitation, making the ICF protocol more robust. The promoted facilitation might represent a form of short-term plasticity, shifting the interplay between facilitation and inhibition, toward facilitation. This might be attributed to temporal summation of excitatory post-synaptic potentials (EPSP) and calcium, due to degree of change in conditioning TMS-MEP amplitudes, compared to single-pulse TMS-MEP amplitudes are marked with \#. $* p<0.05$,

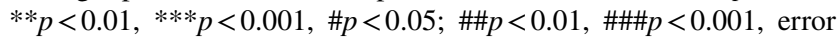
bars indicate SEM

the repetitive CS (Atluri and Regehr 1996; Hennig 2013). According to our results, however, more than 3 CS do not strengthen facilitation any further and do not yield extra benefit. Concerning the role of ISI in ICF protocols, facilitation had been previously described as most prominent with 10, 15, and 25 ms ISI (Lazzaro et al. 2006; Kujirai et al. 1993; Nakamura et al. 1997). Our results are consistent with the literature, as we observed significant facilitation with both 10 and 15 ms ISI. Interestingly, facilitation with $10 \mathrm{~ms}$ was significantly higher, compared to $15 \mathrm{~ms}$ ISI. An ISI of $10 \mathrm{~ms}$, therefore, seems to be more robust to produce facilitation. Similarly, earlier studies have shown facilitation with an ISI of $10 \mathrm{~ms}$ to be more prominent (Hanajima 2009, Wessel 2019). It may also be of interest, whether ICF duration can be prolonged by the increase of CS numbers. In the present study, we did not apply ISIs longer than 15 ms. However, earlier reports using $3 \mathrm{CS}$ have shown significant facilitation with 20-25 ms ISI. Whether this facilitation reflects a prolonged ICF due to EPSP summation, or underlines a different mechanism such as intrinsic rhythm of the motor cortex, is still debated (Hanajima et al. 2009; Hassan et al. 2020; Groiss et al. 2017).

Our results regarding single CS conditions did not show facilitation. This is consistent with the idea that paired pulse TMS protocols in general and the ICF protocol in particular may have a high variability (Caranzano et al. 2017; Hermsen et al. 2016; Orth et al. 2003; Wassermann 2002). However, we cannot exclude that this variability may at least partly be due to interindividual variability for optimal CS intensity to induce ICF. This might focus on the issue that a higher number of CS is more predictable in producing facilitation, than a single CS. Nevertheless, it cannot be entirely ruled out that the lack of facilitation following one CS might be 
due to suboptimal individual CS intensity, which may vary between individuals. Sill, given the fact that previous experiments showed that increasing CS intensity can enhance and prolong facilitation (Orth et al. 2003; Hassan et al. 2020) and at the same time too high CS intensity makes stimulation less selective and also alter the facilitation mechanism, the choice of CS intensity at 90\% AMT may be regarded feasible. It is up to further research to explore facilitation variability on an individual basis while taking into account both number and intensity of CS.

\section{Inhibition (3-4 ms ISI)}

While a train of CS strengthens facilitation, at the same time, it weakens inhibition. In fact, a train of CS might have similar impact on SICI protocols as an increase in CS intensity has. The relation between CS intensity and SICI had been well explored in the past, with SICI strength exhibiting a U-shaped function curve when varying CS intensity (Ilić et al. 2002; Kujirai et al. 1993). Indeed, the MEP amplitude change is believed to be a net outcome of inhibition and facilitation (Ni and Chen 2008), on which CS intensity has modulatory effect (Peurala et al. 2008). Analogously, a train of CS might similarly modulate the inhibition/facilitation interplay, promoting one of the two. While single CS in the range of 3-4 ms ISI causes inhibition because of the here prevailing monosynaptic inhibitory input toward the pyramidal cells, an increasing CS number probably leads EPSP summation, and thus to facilitation.

The argument above is supported by the finding that $4 \mathrm{~ms}$ ISI promoted facilitation with an increasing number of CS. Here, the increased number of CS might entrain EPSPs and reinforce them, as EPSPs are known to occur about 2-5 ms after initial cell depolarization (Curtis and Eccles 1959). Therefore, such summation of EPSP could explain why a train of $7 \mathrm{CS}$ at $4 \mathrm{~ms}$ ISI resulted in significant facilitation, rather than in inhibition. Another explanation why SICI subsided after a train of CS, might be a superimposition of ICF on SICI. If a train of CS is applied at 3-4 ms ISI, then the time between the first CS and the TS would be shifted into the facilitatory ISI window around 10-20 ms.

\section{Outcomes significance}

The reduction of variability is highly important for facilitatory TMS protocols including ICF, as this would improve protocol quality and facilitate comparability between different studies. Different strategies might be used to increase the robustness of the ICF protocol and one possibility would be to increase the CS intensity (Hassan et al. 2020). However, higher CS intensity is thought to active a larger and more heterogeneous neuronal population (Ziemann and Rothwell 2000). Instead, the present study suggests an alternative approach, by increasing the number of CS, which might limit its effects to an activation of a more uniform neuronal population. This hypothesis is supported by our findings, where MEP differed only between 1CS and a train of CS, but not between 3, 5 and 7CS. Therefore, we believe that using a train of CS might be more advantageous than increasing the CS intensity. In this way, the neurons might be targeted more selectively, without the danger of afflicting the physiological mechanism behind the protocol.

Our results could prove advantageous in establishing more robust ICF protocols, while, at the same time, reducing variability and avoiding high TMS intensities. Indeed, variability reported in ICF protocols seems to be a major issue, making ICF less reliable (Hermsen et al. 2016; Orth et al. 2003). Earlier investigation, involving ICF in Parkinson's disease (Bologna et al. 2018; Ni and Chen 2015), dystonia (Berardelli et al. 2008), Alzheimer's disease (Ni and Chen 2015), attention-deficit/hyperactivity disorder (Richter et al. 2007), migraine (Cosentino et al. 2018), as well as sleep research (Doeltgen and Ridding 2010), and rehabilitation medicine (Lulic et al. 2017; Singh et al. 2014) revealed either no ICF difference between control versus condition groups, or showed incongruent outcomes. Thus, ICF protocol reliability might be hampered due to technical reasons, rather than a real lack of facilitatory network difference between patients and controls. Hence, our finding might help to decrease variability when applying ICF protocols and may be useful when investigating facilitatory networks in patients with neurological diseases.

Author contributions 1. Research project: A. conception, B. organization, C. execution; 2. statistical analysis: A. design, B. execution, C. review and critique; 3 . manuscript preparation: $A$. writing of the first draft, B. review and critique. PN and JVZ: 1- BC; 2-B; 3-A. SSH, PA and AS: 1B; 2C; 3B. SJG: 1AB; 2AC; 3B. All authors approved the final version of the manuscript.

Funding Open Access funding enabled and organized by Projekt DEAL.

Data availability Data are available on a reasonable request from the corresponding author.

Code availability Not applicable.

\section{Compliance with ethical standards}

Conflict of interest PN, JVZ, SSH, PA, AS, and SJG have no conflicts of interest to declare in relation to this work.

Ethics approval The study was approved by the local ethics committee and is in accordance with the Declaration of Helsinki. 
Consent to participate All patients provided a written informed consent to participate.

Consent for publication Not applicable.

Open Access This article is licensed under a Creative Commons Attribution 4.0 International License, which permits use, sharing, adaptation, distribution and reproduction in any medium or format, as long as you give appropriate credit to the original author(s) and the source, provide a link to the Creative Commons licence, and indicate if changes were made. The images or other third party material in this article are included in the article's Creative Commons licence, unless indicated otherwise in a credit line to the material. If material is not included in the article's Creative Commons licence and your intended use is not permitted by statutory regulation or exceeds the permitted use, you will need to obtain permission directly from the copyright holder. To view a copy of this licence, visit http://creativecommons.org/licenses/by/4.0/.

\section{References}

Atluri PP, Regehr WG (1996) Determinants of the time course of facilitation at the granule cell to Purkinje cell synapse. J Neurosci 16:5661-5671

Berardelli A, Abbruzzese G, Chen R, Orth M, Ridding MC, Stinear C, Suppa A, Trompetto C, Thompson PD (2008) Consensus paper on short-interval intracortical inhibition and other transcranial magnetic stimulation intracortical paradigms in movement disorders. Brain Stimulat 1:183-191. https://doi. org/10.1016/j.brs.2008.06.005

Bologna M, Guerra A, Paparella G, Giordo L, Alunni Fegatelli D, Vestri AR, Rothwell JC, Berardelli A (2018) Neurophysiological correlates of bradykinesia in Parkinson's disease. Brain 141:2432-2444. https://doi.org/10.1093/brain/awy 155

Calancie B, Wang D, Young E, Alexeeva N (2018) Four-pulse transcranial magnetic stimulation using multiple conditioning inputs normative MEP responses. Exp Brain Res 236:1205-1218. https ://doi.org/10.1007/s00221-018-5212-8

Caranzano L, Stephan MA, Herrmann FR, Benninger DH (2017) Desynchronization does not contribute to intracortical inhibition and facilitation: a paired-pulse paradigm study combined with TST. J Neurophysiol 117:1052-1056. https://doi.org/10.1152/ jn.00381.2016

Cosentino G, Di Marco S, Ferlisi S, Valentino F, Capitano WM, Fierro B, Brighina F (2018) Intracortical facilitation within the migraine motor cortex depends on the stimulation intensity. A paired-pulse TMS study. J. Headache Pain 19:65. https://doi. org/10.1186/s10194-018-0897-4

Curtis DR, Eccles JC (1959) The time courses of excitatory and inhibitory synaptic actions. J Physiol 145:529-546. https://doi. org/10.1113/jphysiol.1959.sp006159

Doeltgen SH, Ridding MC (2010) Behavioural exposure and sleep do not modify corticospinal and intracortical excitability in the human motor system. Clin Neurophysiol 121:448-452. https:// doi.org/10.1016/j.clinph.2009.11.085

Doruk Camsari D, Lewis CP, Sonmez AI, Nandakumar AL, Gresbrink MA, Daskalakis ZJ, Croarkin PE (2019) Transcranial magnetic stimulation markers of antidepressant treatment in adolescents with major depressive disorder. Int J Neuropsychopharmacol 22:435-444. https://doi.org/10.1093/ijnp/pyz021

Groiss SJ, Mochizuki H, Hanajima R, Trenado C, Nakatani-Enomoto S, Otani K, Ugawa Y (2017) Impairment of triad conditioned facilitation in amyotrophic lateral sclerosis. Amyotroph Lateral
Scler Frontotemporal Degener 18(7-8):604-610. https://doi. org/10.1080/21678421.2017.1321676

Hanajima R, Terao Y, Hamada M, Okabe S, Nakatani-Enomoto S, Furubayashi T, Yugeta A, Inomata-Terada S, Ugawa Y (2009) Forty-hertz triple-pulse stimulation induces motor cortical facilitation in humans. Brain Res 1296:15-23. https://doi. org/10.1016/j.brainres.2009.08.008

Hassan SS, Trenado C, Rageh TA, Schnitzler A, Groiss SJ (2020) Effect of conditioning and test stimulus intensity on cortical excitability using triad-conditioning transcranial magnetic stimulation. Exp Brain Res 238:1305-1309. https://doi.org/10.1007/ s00221-020-05812-z

Hennig MH (2013) Theoretical models of synaptic short term plasticity. Front Comput Neurosci 7:154. https://doi.org/10.3389/ fncom.2013.00154

Hermsen AM, Haag A, Duddek C, Balkenhol K, Bugiel H, Bauer S, Mylius V, Menzler K, Rosenow F (2016) Test-retest reliability of single and paired pulse transcranial magnetic stimulation parameters in healthy subjects. J Neurol Sci 362:209-216. https ://doi.org/10.1016/j.jns.2016.01.039

Ilić TV, Meintzschel F, Cleff U, Ruge D, Kessler KR, Ziemann U (2002) Short-interval paired-pulse inhibition and facilitation of human motor cortex: the dimension of stimulus intensity. J Physiol 545:153-167. https://doi.org/10.1113/jphys iol.2002.030122

Kujirai T, Caramia MD, Rothwell JC, Day BL, Thompson PD, Ferbert A, Wroe S, Asselman P, Marsden CD (1993) Corticocortical inhibition in human motor cortex. J Physiol 471:501-519

Lazzaro VD, Pilato F, Dileone M, Ranieri F, Ricci V, Profice P, Bria P, Tonali PA, Ziemann U (2006b) GABAA receptor subtype specific enhancement of inhibition in human motor cortex. J Physiol 575:721-726. https://doi.org/10.1113/jphys iol.2006.114694

LazzaroOliviero VDA, Pilato F, Saturno E, Dileone M, Mazzone P, Insola A, Tonali PA, Rothwell JC (2004) The physiological basis of transcranial motor cortex stimulation in conscious humans. Clin Neurophysiol 115:255-266. https://doi.org/10.1016/j.clinp h.2003.10.009

LazzaroPilato VDF, Oliviero A, Dileone M, Saturno E, Mazzone P, Insola A, Profice P, Ranieri F, Capone F, Tonali PA, Rothwell JC (2006) Origin of facilitation of motor-evoked potentials after paired magnetic stimulation: direct recording of epidural activity in conscious humans. J Neurophysiol 96:1765-1771. https://doi. org/10.1152/jn.00360.2006

LazzaroRothwell VDJC, Oliviero A, Profice P, Insola A, Mazzone P, Tonali P (1999) Intracortical origin of the short latency facilitation produced by pairs of threshold magnetic stimuli applied to human motor cortex. Exp Brain Res 129:494-499. https://doi. org/10.1007/s002210050919

Lulic T, El-Sayes J, Fassett HJ, Nelson AJ (2017) Physical activity levels determine exercise-induced changes in brain excitability. PLoS ONE 12:e0173672. https://doi.org/10.1371/journal.pone.0173672

Nakamura H, Kitagawa H, Kawaguchi Y, Tsuji H (1997) Intracortical facilitation and inhibition after transcranial magnetic stimulation in conscious humans. J Physiol 498:817-823. https://doi. org/10.1113/jphysiol.1997.sp021905

Ni Z, Chen R (2008) Short-interval intracortical inhibition: a complex measure. Clin Neurophysiol 119:2175-2176. https://doi. org/10.1016/j.clinph.2008.06.007

Ni Z, Chen R (2015) Transcranial magnetic stimulation to understand pathophysiology and as potential treatment for neurodegenerative diseases. Transl Neurodegener 4:22. https://doi.org/10.1186/ s40035-015-0045-x

Orth M, Snijders AH, Rothwell JC (2003) The variability of intracortical inhibition and facilitation. Clin Neurophysiol 114:2362-2369. https://doi.org/10.1016/s1388-2457(03)00243-8 
Peurala SH, Müller-Dahlhaus JFM, Arai N, Ziemann U (2008) Interference of short-interval intracortical inhibition (SICI) and shortinterval intracortical facilitation (SICF). Clin Neurophysiol 119:2291-2297. https://doi.org/10.1016/j.clinph.2008.05.031

Richter MM, Ehlis A-C, Jacob CP, Fallgatter AJ (2007) Cortical excitability in adult patients with attention-deficit/hyperactivity disorder (ADHD). Neurosci Lett 419:137-141. https://doi.org/10.1016/j. neulet.2007.04.024

Rossini PM, Burke D, Chen R, Cohen LG, Daskalakis Z, Di Iorio R, Di Lazzaro V, Ferreri F, Fitzgerald PB, George MS, Hallett M, Lefaucheur JP, Langguth B, Matsumoto H, Miniussi C, Nitsche MA, Pascual-Leone A, Paulus W, Rossi S, Rothwell JC, Siebner HR, Ugawa Y, Walsh V, Ziemann U (2015) Non-invasive electrical and magnetic stimulation of the brain, spinal cord, roots and peripheral nerves: Basic principles and procedures for routine clinical and research application. An updated report from an I.F.C.N. Committee Clin Neurophysiol 126:1071-1107. https://doi.org/10.1016/j. clinph.2015.02.001

Rothwell JC (1997) Techniques and mechanisms of action of transcranial stimulation of the human motor cortex. J Neurosci Methods 74:113-122. https://doi.org/10.1016/S0165-0270(97)02242-5

Singh AM, Duncan RE, Neva JL, Staines WR (2014) Aerobic exercise modulates intracortical inhibition and facilitation in a nonexercised upper limb muscle. BMC Sports Sci Med Rehabil 6:23. https://doi.org/10.1186/2052-1847-6-23

Wessel MJ, Draaisma LR, Morishita T, Hummel FC (2019) The effects of stimulator, waveform, and current direction on intracortical inhibition and facilitation: a TMS comparison study. Front Neurosci 13:703. https://doi.org/10.3389/fnins.2019.00703

Wassermann EM (2002) Variation in the response to transcranial magnetic brain stimulation in the general population. Clin Neurophysiol Off J Int Fed Clin Neurophysiol 113:1165-1171. https ://doi.org/10.1016/s1388-2457(02)00144-x

World Medical Association (2013) World Medical Association Declaration of Helsinki: ethical principles for medical research involving human subjects. JAMA 310:2191-2194. https://doi. org/10.1001/jama.2013.281053

Ziemann U, Rothwell JC (2000) I-waves in motor cortex. J Clin Neurophysiol 17:397-405. https://doi.org/10.1097/00004691-20000 7000-00005

Publisher's Note Springer Nature remains neutral with regard to jurisdictional claims in published maps and institutional affiliations. 